\title{
The role of palladium dynamics in surface catalysis of coupling reactions
}

\author{
Lidong Shao, Bingsen Zhang, Wei Zhang, Sung You Hong, Robert Schlögl and Dang Sheng Su*
}

Ever since its discovery, the carbon-carbon cross coupling reaction has contributed greatly to build up molecules with chemical complexity accessible, which is crucial for organic synthesis and pharmaceutical drug development. ${ }^{[1-4]}$ Surface catalyzing carboncarbon bond formation on supported Pd nanoparticles (PdNPs) ${ }^{[5-8]}$ can reduce product contamination and aid in the development of green chemistry through organic synthesis. On the nanoscale, unlike in the bulk phase, PdNPs exhibit a large specific surface area and abundant low coordination sites that enable increased catalytic activity. However, the catalytic reaction mechanisms on supported PdNPs surfaces remain a topic of controversial discussion. Some reports $^{[9-11]}$ suggest that surface sites on PdNPs may play a crucial role in catalyzing coupling reactions, while others propose that the Pd species leached from support into solution govern the reaction pathways. $^{[12,13]}$

As a conventional approach to interpret catalysis properties, surface analyses of supported PdNPs used during cross coupling reactions have been rarely examined due to technical difficulties in observing surfaces of nanoparticles with atomic precision ${ }^{[14,15]}$ Recent work suggests that PdNPs smaller than $1 \mathrm{~nm}$ are commonly missed during most microscopic studies. ${ }^{[14]}$ In addition, surface analysis of electronic properties of reacted PdNPs, is complicated by the fact that signal collection on highly dispersed particles is challenging due to the geometric and electric interference on bulk supports after cross-coupling reactions. ${ }^{[15]}$

In present work, we set out to firstly investigate the catalytic performance of PdNPs supported on materials with various levels of functionalities in the context of Suzuki-Miyaura reaction. Secondly, Pd dynamics was investigated in terms of surface changes on PdNPs and support for understanding obtained catalytic results. Unlike previous reports applying active carbon, ${ }^{[16,17]}$ silica, ${ }^{[18,19]}$ alumina, ${ }^{[20,21]}$ or zeolites ${ }^{[22,23]}$ in heterogeneous catalysis of coupling reaction, the current work exploits functionalized CNTs as support. To study the influence of chemical properties of supporting materials on Pd dynamics, CNTs from a same source of production

[*] Dr. Dang Sheng Su, Dr. Lidong Shao, Dr. Bingsen Zhang, Dr. Wei Zhang, Prof. Dr. Robert Schlögl Department of Inorganic Chemistry, Fritz Haber Institute of the Max Planck Society, Faradayweg 4-6, 14195 Berlin, Germany

Fax: (+49) 3084134401

E-mail: dangsheng@fhi-berlin.mpg.de Homepage: www.fhi-berlin.mpg.de

Dr. Bingsen Zhang, Dr. Dang Sheng Su

Shenyang National Laboratory for Materials Science, 72 Wenhua Road, 110016 Shenyang, China

\section{Dr. Sung You Hong}

School of Nano-Bioscience and Chemical Engineering, Ulsan National Institute of Science and Technology, Ulsan 689-798, Republic of Korea.

$[* *] \quad$ The authors acknowledge the support from the EnerChem Project at the Fritz Haber Institute of the Max Planck Society. We are also grateful for the experimental support from Professor Peter $\mathrm{H}$. Seeberger.

Supporting information for this article is available on the WWW under http://www.angewandte.org or from the author. with different graphitic degrees were functionalized under same conditions and applied for supporting PdNPs in coupling reactions.
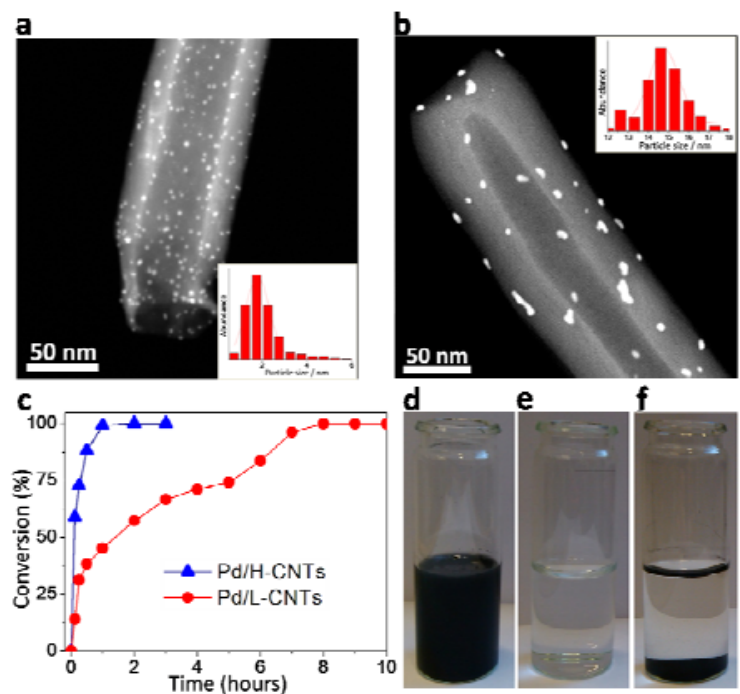

Figure 1 Microscopic image of PdNPs on CNTs, conversion versus reaction time, and pictures of reaction solutions. a, scanning transmission electron (STEM) image of Pd/H-CNTs. b, STEM image of Pd/L-CNTs. c, Conversion of Suzuki-Miyaura reactions as a function of reaction time when using $\mathrm{Pd} / \mathrm{H}-\mathrm{CNTs}$ and $\mathrm{Pd} / \mathrm{L}-\mathrm{CNTs}$ as catalysts. Reactants of iodobenzene, phenylboronic acid, together with $\mathrm{K}_{2} \mathrm{CO}_{3}$ were heated to $60^{\circ} \mathrm{C}$ in a mixture of water and DMF (1:1). d, Solution image of $\mathrm{Pd} / \mathrm{H}-$ CNTs after 1 hour reaction and 10 minutes standing. d, Filtrate of the solution in Figure 1d. f, Solution image of Pd/L-CNTs after 1 hour reaction and 10 minutes standing.

After CNT growth, it underwent annealing treatments at $700^{\circ} \mathrm{C}$ and $1500^{\circ} \mathrm{C}$ to remove the polyaromatic carbon and graphitized as-grown carbon structures. ${ }^{[24]}$ As for functionalization step, CNTs were added into concentrated nitric acid with vigorous stirring at $120^{\circ} \mathrm{C}$. Because CNTs annealed at $700^{\circ} \mathrm{C}$ possess more defective vacancies than CNTs annealed at $1500^{\circ} \mathrm{C}, \mathrm{HNO}_{3}$ treatment introduced a high functionalization (H-CNTs) on defective CNTs and a low functionalization (L-CNTs) on graphitized CNTs. Functionalities act as entrapping sites for anchoring metal cations during impregnation. ${ }^{[25]}$ Vacancies and cavities of CNTs may also play roles in entrapping foreign species. ${ }^{[26]}$ In current work, figure 1a illustrates the dispersion of PdNPs ( $2 \%$ wt palladium) on $\mathrm{H}-$ CNTs (Pd/H-CNTs) with a size distribution of $1.8 \pm 0.2 \mathrm{~nm}$ using STEM mode. Figure 1b displays a poor dispersion of PdNPs (2\% wt palladium) on L-CNTs (Pd/L-CNTs) with a larger size distribution of $14.5 \pm 0.2 \mathrm{~nm}$. Catalytic properties of reactant conversion versus reaction time are shown in Figure 1c. Quantitative conversion was obtained within one hour when $\mathrm{Pd} / \mathrm{H}-\mathrm{CNT}$ s was used, while reactions with $\mathrm{Pd} / \mathrm{L}-\mathrm{CNT}$ s required 8 hours to reach quantitative conversion. The turn over frequency (TOF) of completing reaction is $990 \mathrm{~h}^{-1}$ for $\mathrm{Pd} / \mathrm{H}-\mathrm{CNTs}$ and $124 \mathrm{~h}^{-1}$ for Pd/L-CNTs. Figure $1 \mathrm{~d}$ shows the Suzuki-Miyaura reaction solution of Pd/H-CNTs after one hour reaction time. This black solution could stand still for 10 minutes with no precipitate was observed. Further filtration using normal filter papers collected all the $\mathrm{Pd} / \mathrm{H}-\mathrm{CNTs}$ and result in a clear solution (Figure 1e). As for reaction solution of Pd/L-CNTs 
after one hour reaction time, it precipitated completely after 10 minutes standing, as seen in Figure 1f.

Significant difference in obtained reactivity prompted a detailed analysis of the catalysts status. Electron microscope was applied to probe the state of the CNT-supported catalysts. Reactions were stopped at 1 hour for Pd/H-CNTs and Pd/L-CNTs. With the majority H-CNTs remaining intact (Figure S1), some H-CNTs with graphitic structure damage were observed after catalyzing coupling reaction. Herein, we show such morphology changed Pd/H-CNTs in Figure 2a and Figure 2b. A STEM image in Figure 2a displays the dispersion status of PdNPs on a structure damaged H-CNT. While some PdNPs exhibit a larger size distribution, PdNPs with a diameter of 1-2 nm are still visible. As for the information on $\mathrm{H}$ CNTs after reaction, TEM was applied for a local view of the damage status, as seen in Figure 2b. Tracks of "PdNPs movement" can be observed on H-CNTs. XRD analyses (Figure S2) are consistent with microscopic studies, as the carbon structure changes shown in Figure 2 occurred to some H-CNTs, but not to the majority. As for the one hour reacted $\mathrm{Pd} / \mathrm{L}-\mathrm{CNT}$, although reaction was not completed, obvious leaching of PdNPs from the outer walls of LCNTs was observed, and PdNPs inside L-CNT channels sintered into larger aggregates (Figure 2c, d). In addition, no damage to the graphitic structure L-CNTs was observed.

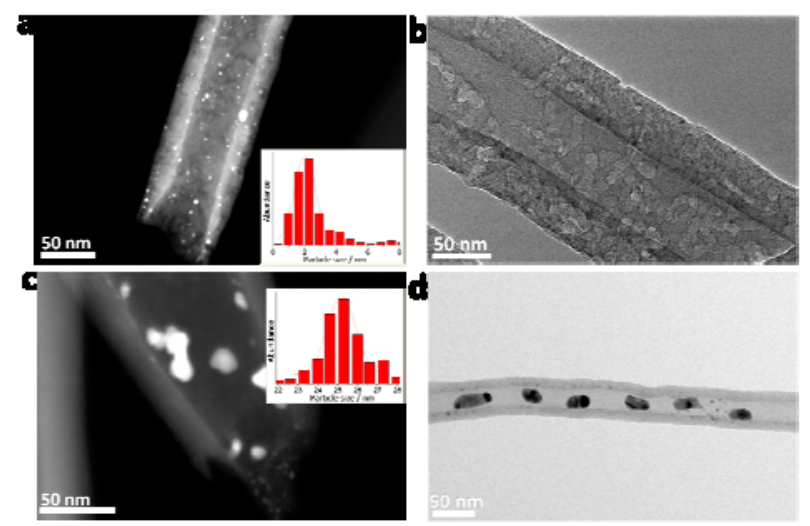

Figure 2 Microscopic observations of Pd/H-CNTs and Pd/LCNTs after 1 hour catalyzations. a, STEM image of Pd/H-CNTs. b, Transmission electron microscope (TEM) image of $\mathrm{Pd} / \mathrm{H}$ CNTs. c, STEM image of Pd/L-CNTs. d, TEM image of Pd/LCNTs.

The morphological changes on reacted $\mathrm{Pd} / \mathrm{H}-\mathrm{CNTs}$ are remarkable, as illustrated by the moving tracks of catalyticallyactive PdNPs on H-CNTs. Visual evidence of particle moving tracks on supporting materials has not been reported to date. Our evidence may provide precious information about the dynamics of catalytically-active species under reaction conditions, thus contributing to a better understanding of catalysis pathways. Moreover, the well-known physical and mechanical properties of CNTs indirectly demonstrate the magnitude of the observed dynamic took place in the current work. Standard CNTs can withstand a pressure up to $24 \mathrm{GPa}$ without deformation. Bulk modulus studies revealed that CNTs composed of a hard phase can withstand a pressure even higher than diamond. ${ }^{[27]}$ Engraving and forming channels on CNTs vividly reveals the reaction-driven migrations of PdNPs. Microscopic results indicate a strong metalsupport interaction on $\mathrm{H}$-CNTs and are consistent with previous studies that PdNPs leached from unmodified inorganic substrates precipitate in aggregates during coupling reactions. ${ }^{[28,29]}$

The morphological changes on supporting H-CNTs drew our attention, requiring a more in-depth examination of the surface and crystalline structure of the supported PdNPs. As-prepared $\mathrm{Pd} / \mathrm{H}$ CNTs and $\mathrm{Pd} / \mathrm{L}-\mathrm{CNTs}$ possess metallic phases of $\mathrm{Pd}$ before catalyzing the coupling reaction. Unlike reacted $\mathrm{Pd} / \mathrm{L}-\mathrm{CNT}$ semains in the Pd metallic phase (Figure 3a), Pd/H-CNTs after one hour catalyzation (Figure $3 b$ ) displays a crystalline change in near surface

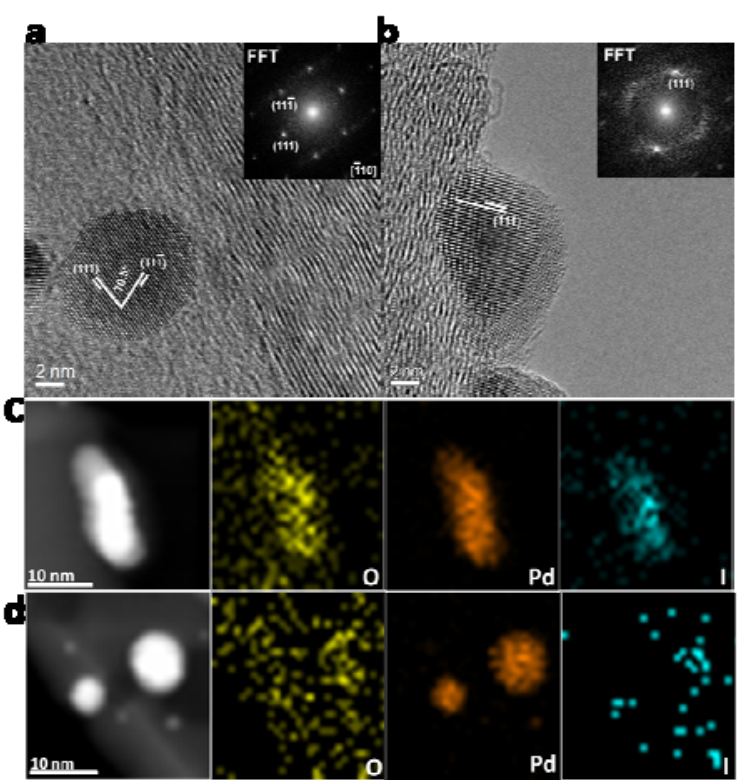

Figure 3 High resolution microscopic observations of $\mathrm{Pd} / \mathrm{H}-\mathrm{CNT}$ and Pd/L-CNTs after 1 hour catalyzation. a, high-resolution TEM (HRTEM) image of a Pd nanoparticle on L-CNTs. b, HRTEM image of a Pd nanoparticle on H-CNTs. c, Energy dispersion Xray in the STEM mode (STEM-EDX) maps of a Pd nanoparticle supported on H-CNTs. d, STEM-EDX maps of a Pd nanoparticle supported on L-CNTs.

regions of PdNPs in HRTEM analyses. While distorted crystalline phases were observed in the near surface regions, the cores of these PdNPs remained as palladium metallic phases. Clear boundaries between the metallic cores and the distorted crystalline surfaces indicate that phase changes occurred on the Pd surfaces during the coupling reaction. Crystalline changes took place on PdNPs, which are supported on both damaged and structure intact H-CNTs. The present observation of surface changes on supported PdNPs was surprising as aggregated Pd is almost exclusively obtained after Suzuki-Miyaura reactions in both homogeneous and heterogeneous systems. $^{[8,15,30,31]}$ To date, no direct visual evidence for surface changes associated with the "core-shell" structure of the Pd metallic phase (core) and distorted surface (shell) has been reported. This observation indicates that surface changes had taken place on PdNPs that remained entrapped on H-CNTs. Re-adsorption of PdNPs after the reaction is possible, but generally forms Pd aggregate. Even if PdNPs re-adsorb prior to the completion of a coupling reaction, no nanoparticles with "core-shell" structures would result. Because on a re-adsorbed Pd nanoparticle, the distorted surface (wrapping-shell) would have been present between the core and H-CNTs. Thus, a metallic Pd core would have no chance to directly contact the $\mathrm{H}$ CNTs surface as observed here.

Significant crystalline changes (Figure $3 b$ ) indicate a modification of elemental compositions on supported PdNPs during catalyzation. Maps of a Pd nanoparticle supported on H-CNTs using a STEM-EDX mode (Figure 3c) after catalyzing for 1 hour exhibit homogeneous distributions of $\mathrm{O}$ and Pd elements. More importantly, an iodine signal was clearly traced on the Pd particle. In contrast, maps for a Pd nanoparticle on L-CNTs after a 1 hour reaction show elemental signals with significantly reduced intensities (Figure 3d). In line with HRTEM analyses of metallic phase Pd on L-CNTs and the distorted crystalline phase of Pd on H-CNTs, contrasting oxygen intensities in elemental maps indicate an appearance of oxidativelyadded complexes on reacted PdNPs on H-CNTs. A trace of reactive intermediates has long been pursued in the form of oxidativelyadded complexes. ${ }^{[8,15]}$ The consistent occurrences of crystalline changes and iodine trace suggest intermediate species resulting from oxidative addition into a C(aryl)-I bond.

A mechanism sketch is shown in Figure 4, which is based on analytical evidences in current work. Added to water, H-CNTs 
possess an enriched surface charge, and they repulse each other in an aqueous solution to form a stabilized CNT dispersion. In the course of Suzuki-Miyaura reactions, the oxidative addition step requires the participation of Pd nanospecies and possibly generates leaching in the form of Pd aggregates. In our work, we confirmed that most of PdNPs were entrapped on H-CNT due to the inherent stability of the PdNPs on functionalized CNTs under reaction conditions. As shown in Figure 4, reactant molecules co-dispersed with $\mathrm{Pd} / \mathrm{H}-\mathrm{CNTs}$ and adsorbed on embedded PdNPs with high surface areas, thus enhancing the possibility of surface catalysis on supported PdNPs. Crystalline changes of PdNPs were presently observed on H-CNTs with and without graphitic structural damage. Structural damaged (engraving graphitic walls) H-CNTs could be caused by the catalyst preparation, in which PdNPs loading can be slightly varied due to the distribution of functionality on H-CNTs. Thus, a higher PdNPs loading on a small diameter H-CNT with thin (less graphitic layers) multi-walls could result in a stronger dynamics on PdNPs and corresponding damages on H-CNTs during catalyzing reactions. Although leaching became possible in this circumstance, the engraved CNTs structures displayed a high magnitude of driven force on dynamic PdNPs against metal-support interactions, and thus revealed catalysis tracks with optical evidences.

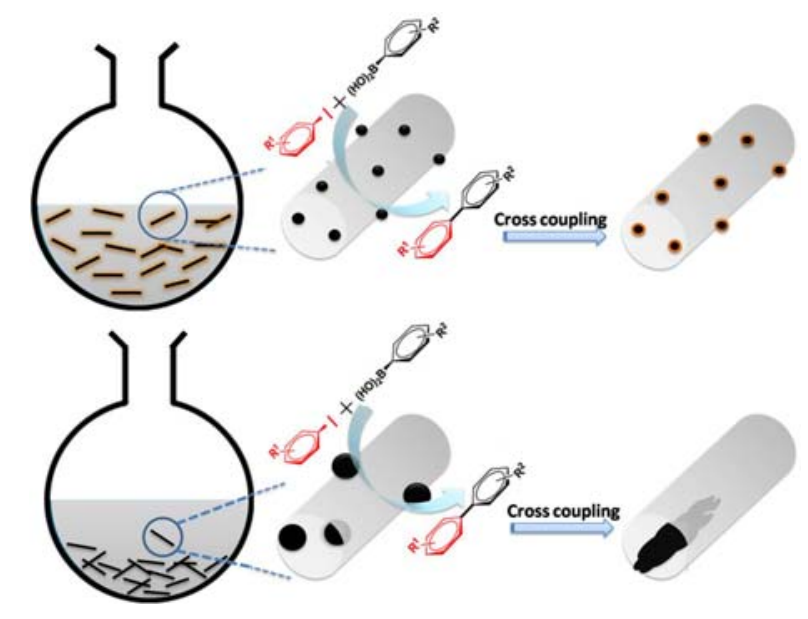

Figure 4 A scheme of proposed reaction mechanism based on analytical evidences for Pd/H-CNTs and Pd/L-CNTs during catalyzations.

In this work, functionality and dispersibility of supporting materials were exploited to alter the metal-support interaction between PdNPs and functionalized CNTs. Thus, the role of Pd dynamics in surface catalysis of coupling reactions can be studied. The observed high reactivity did not lead to obvious leaching of PdNPs during the Suzuki-Miyaura reaction, however, it resulted in surface changes on PdNPs and supported H-CNTs. Corresponding to commonly-reported high reactivity in homogeneous catalysis, carbon-carbon couplings with high efficiency can be achieved on supported PdNPs by improving surface functionalization and dispersibility of the catalyst. Such a system, in addition, offers opportunities for characterization with atomic precision for tracking surface catalysis, which is crucial for detecting dynamic changes on catalytically-active species and understanding catalysis pathways. The presently discussed approach may also be applicable to systems using other inorganic substrates for supporting PdNPs. Future work will focus on modifying supporting materials with various surface properties for the interest of studying the role of Pd dynamics in surface catalysis.

Methods

Synthesis and materials. For experimental details of CNTs functionalization and Pd loading, see the Supporting Information. employed to conduct structural investigations. For more experimental details of electron microscopy, see the Supporting Information.

Catalysis. All chemicals were reagent grade and used as supplied except where noted. For more experimental details, please consult the Supporting Information.

Received: ((will be filled in by the editorial staff))

Published online on ((will be filled in by the editorial staff))

Keywords: ·Pd nanoparticle, surface catalysis, coupling reaction ·

[1] A. Suzuki, N. Miyaura, J. Chem. Soc., Chem. Commun, 1979, 866.

[2] A. Zapf, M. Beller, Top. Catal. 2002, 19, 101.

[3] N. Miyaura, A. Suzuki, Chem. Rev. 1995, 95, 2457.

[4] A. Suzuki, J. Organomet. Chem. 1999, 576, 147.

[5] L. Djakovitch, K. Koehler, J. Am. Chem. Soc. 2001, 123, 5990.

[6] A. F. Schmidt, V. V. Smirnov, J. Mol. Catal. A. 2003, 203, 75.

[7] A. F. Schmidt, V. V. Smirnov, Top. Catal. 2005, 32, 71.

[8] M. T. Reetz, E. Westermann, Angew. Chem. Int. Ed. 2000, 39, 165

[9] J. J. Davis, K. S. Coleman, K. L. Busuttil, C. B. Bagshaw, J. Am. Chem. Soc. 2005, 127, 13082

[10] J. J. Davis, C. B. Bagshaw, K. L. Busuttil, Y. Hanyu, K. S. Coleman, J. Am. Chem. Soc. 2006, 128, 14135.

[11] P. J. Ellis, I. J. S. Fairlamb, S. F. J. Hackett, K. Wilson, A. F. Lee, Angew. Chem. Int. Ed. 2010, 49, 1820.

[12] T. S. N. Phan, M. Van der Sluys, C. W. Jones, Adv. Synth. Catal. 2006, 348, 609.

[13] C. C. Cassol, A. P. Umpierre, G. Machado, S. I. Wolke, J. Dupont, J. Am. Chem. Soc. 2005, 127, 3298.

[14] B. S. Zhang, W. Zhang, D. S. Su, ChemCatChem. 2011, 3, 965.

[15] S. MacQuarrie, J. H. Horton, J. Barnes, K. McEleney, H. P. Loock, C. M. Crudden, Angew. Chem. Int. Ed. 2008, 47, 3279.

[16] H. Sakurai, T. Tsukuda, T. Hirao, J. Org. Chem. 2002, 67, 2721.

[17] R. K. Arvela, N. E. Leadbeater, Org. Lett. 2005, 7, 2101.

[18] R. B. Bedford, U. G. Singh, R. I. Walton, R. T. Williams, S. A. Davis, Chem. Mater. 2005, 17, 701.

[19] N. Erathodiyil, S. Ooi, A. M. Seayad, Y. Han, S. S. Lee, J. Y. Ying, Chem. Eur. J. 2008, 14, 3118.

[20] R. L. Augustine, S. T. O’Leary, J. Mol. Catal. 1992, 72, 229.

[21] A. Biffis, M. Zecca, M. Basato, Eur. J. Inorg. Chem. 2001, 1131.

[22] L. Artok, H. bulut, Tetra. Lett. 2004, 45, 3881.

[23] M. Dams, L. Drijkoningen, B. Pauwels, G. Van Tendeloo, D. E. De Vos, P. A. Jacobs, J. Catal. 2002, 209, 225.

[24] J. P. Tessonnier, D. Rosenthal, T. W. Hansen, C. Hess, O. Timpe, D. S. Su, Carbon. 2009, 47, 1779.

[25] L. D. Shao, W. Zhang, M. Armbrüster, D. Teschner, F. Girgsdies, B. S. Zhang, O. Timpe, M. Friedrich, R. Schlögl, D. S. Su, Angew. Chem. Inter. Ed. 2011, 50, 10231. Angew. Chem. 2011, 123 , 10414.

[26] L. D. Shao, T.-W. Lin, G. Tobias, M. L. H. Green, Chem. Commun. 2008, 2164.

[27] M. Popov, M. Kyotani, R. Nemanich, Y. Koga, Phys. Rev. B. 2002, 65, 033408.

[28] K. Kohler, W. Kleist, S. S. Prockl, Inorg. Chem. 2007, 46, 1876.

[29] F. Y. Zhao, M. Shirai, Y. Ikushima, M. Arai, J. Mol. Catal. A. 2002, 180, 211.

[30] M. T. Reetz, J. G. De Vries, Chem. Commun. 2004, 1559.

[31] J. G. De Vries, Dalton Trans. 2006, 421. 


\section{Surface Catalysis}

Lidong Shao, Bingsen Zhang, Wei Zhang, Sung You Hong, Robert

Schlögl and DangSheng Su*

\section{Page - Page}

The role of palladium dynamics in surface catalysis of coupling reactions

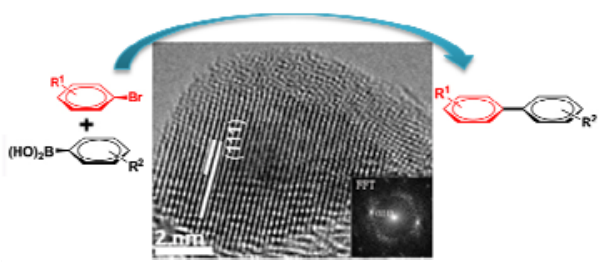

Catalysis mechanism on supported Pd nanoparticles (PdNPs) for cross coupling reactions remains debatable. Here, we study dynamic changes on supported PdNPs during the coupling reaction by exploiting modified carbon nanotubes (CNTs) as supporting materials. Dispersible Pd-CNTs catalyst can co-disperse with reactant in the reaction solution, increase molecular contact and improve reactivity in surface catalysis. CNTs with engraved walls, PdNPs with surface crystalline distortion and elemental trace of iodine were discovered with nanoscale precision for revealing catalysis tracks. 